\title{
Antidepressants might work for people with major depression: where do we go from here? - Author's reply
}

Andrea Cipriani ${ }^{1 *}$, Nicoletta Adamo ${ }^{2}$, Cinzia Del Giovane ${ }^{3}$, David Coghill ${ }^{4,5,6}$, Tobias Banaschewski $^{7}$, Chris Hollis ${ }^{8,9}$, Alessandro Zuddas ${ }^{10}$, Emily Simonoff ${ }^{2}$, Samuele Cortese $e^{8,11,12,13}$

${ }^{1}$ Department of Psychiatry, University of Oxford, and Oxford Health NHS Foundation Trust, Warneford Hospital, Oxford OX3 7JX, UK

${ }^{2}$ Department of Child and Adolescent Psychiatry, King's College London, and Institute of Psychiatry, Psychology and Neuroscience, and National Institute for Health Research (NIHR) Maudsley Biomedical Research Centre, London, UK

${ }^{3}$ Statistics and Methodology, Institute of Primary Health Care (BIHAM), University of Bern, Bern, Switzerland

${ }^{4}$ Departments of Paediatrics and Psychiatry, Faculty of Medicine, Dentistry and Health Sciences, University of Melbourne, Melbourne, VIC, Australia

${ }^{5}$ Division of Neuroscience, Ninewells Hospital and Medical School, University of Dundee, Dundee, UK ${ }^{6}$ Murdoch Childrens' Research Institute, Melbourne, VIC, Australia

${ }^{7}$ Department of Child and Adolescent Psychiatry and Psychotherapy, Central Institute of Mental Health, Medical Faculty Mannheim and University of Heidelberg, Mannheim, Germany

${ }^{8}$ Division of Psychiatry and Applied Psychology, School of Medicine, University of Nottingham, Nottingham, UK

${ }^{9} \mathrm{NIHR}$ MindTech MedTech and In-vitro Diagnostic Cooperative, and Centre for ADHD and Neurodevelopmental Disorders Across the Lifespan (CANDAL), Institute of Mental Health, University of Nottingham, Nottingham, UK

${ }^{10}$ Child and Adolescent Neuropsychiatry Unit, Department of Biomedical Sciences, University of Cagliari and "A Cao" Paediatric Hospital, Cagliari, Italy

${ }^{11}$ Centre for Innovation in Mental Health, Academic Unit of Psychology, and Clinical and Experimental Sciences (CNS and Psychiatry), Faculty of Medicine, University of Southampton, Southampton, UK

${ }^{12}$ Solent NHS Trust, Southampton, UK

${ }^{13}$ New York University Child Study Center, New York, NY, USA

*corresponding author: andrea.cipriani@psych.ox.ac.uk

We agree with Erlend Faltinsen and colleagues that standardised mean differences can be difficult to translate into clinical practice. As reported in the Cochrane handbook, the mean difference (or more correctly, difference in means) measures the absolute difference between the mean value in two groups and then estimates the average amount that the experimental intervention changes in the outcome compared with that of the control intervention. Mean difference can be used in metaanalysis as a summary statistic only when outcome measurements in all studies are made on the 
same scale. By contrast with standardised mean differences, the overall intervention effect can be difficult to interpret because it is reported in units of SD rather than in units of a specific rating scale. Although, in some circumstances, it is possible to transform the effect back to the units used in a specific study, the problem with standardised mean differences is that this method assumes that differences in SD between studies reflect differences in measurement scales and not real differences in variability among study populations. This assumption could be problematic in circumstances in which there might be real differences in variability between the participants in different studies (for instance, pragmatic vs explanatory studies). For this reason, we paid careful attention when we drafted the inclusion and exclusion criteria in the protocol of our review ${ }^{1,2}$ and selected only trials that were similar in design, population, and interventions to reduce heterogeneity and inconsistency. ${ }^{3}$ This selection led to the inevitable exclusion of several trials. Even though we did an extensive search for published and unpublished data and contacted all study authors and pharmaceutical companies for additional data, we might, as is typically the case in systematic reviews, have missed some relevant studies. However, we do not agree that we should have included all the studies in the 2015 Cochrane review. ${ }^{4}$ Before finalising our list of included studies, we screened existing systematic reviews for any relevant reference in their lists of included (and excluded) studies. As detailed in the appendix of our review, we had to exclude several studies that were included by Ole Jakob Storeb $\varnothing$ and colleagues: ${ }^{4} 51$ studies with less than 7 days of treatment, 38 crossover studies without washout period and no pre-crossover data (even after contacting the authors), 18 studies in which patients were responders to previous treatment, 14 studies where treatment was not as monotherapy, and a range of other studies without appropriate randomisation, with single-blind design, that included preschool children, or that administered nonoral formulation of the investigational drug. Including these trials would have been a clear violation of our published protocol and a material risk for the transitivity of the network. ${ }^{3}$

As prespecified in our peer-reviewed protocol, ${ }^{1,2}$ tolerability (proportion of patients who dropped out of studies because of side-effects) was chosen as primary outcome because it is consistently reported across studies and it is a hard outcome used in other similar reviews. 5 We also analysed allcause discontinuation as a pre-defined secondary outcome. It is an important measure of treatment acceptability and full results are reported in the main text of our review and in the online appendix.

We did not include edivoxetine because, when we drafted the protocol, we focused only on the drugs that were licensed or mentioned in international clinical guidelines at the time. We agree with Shuai Wang and Yi Zheng that systematic reviews should be as comprehensive as possible. We are aware that many new drugs for attention deficit hyperactivity disorder (ADHD) will be on the market in the near future. As we did with another network meta-analysis, 6 we plan to publish the update of this review in a few years' time and will include in the network, as appropriate, all the relevant medications that will be available at that time.

In our network meta-analysis, we summarised the best available evidence about efficacy and acceptability of ADHD medications. In the protocol, we planned analyses of clinical outcomes at different time-points (acute and long term) but, unfortunately, there are not enough randomised controlled trials in the field. More long-term data and higher quality studies are urgently needed. We totally agree with John Warren that it is important to consider reliable information also about safety and harms when choosing a pharmacological treatment for ADHD (of course, this applies to any intervention in any disorder in any field of medicine). We are working on this question and have almost completed the data collection for a parallel project (based on the same protocol), which investigates the profile of specific adverse events for each drug, including-among otherspsychotic symptoms, suicidality, sleep problems, headache, loss of appetite, and tics. 
This information about tolerability will complete the clinical picture of the safety profile of ADHD medications and will better inform patients, carers, clinicians, and treatment guidelines.

SC declares reimbursement for travel and accommodation expenses from the Association for Child and Adolescent Central Health (ACAMH) in relation to lectures delivered for $C A M H$, and from Healthcare Convention for educational activity on ADHD. NA declares travel support to attend a conference by Shire. TB declares advisory or consultancy roles for Actelion, Hexal Pharma, Eli Lilly, Medice, Novartis, Oxford Outcomes, Otsuka, PCM Scientific, Shire, and Viforpharma; conference support or speaker's fees from Medice, Novartis, and Shire; royalties from Hogrefe, Kohlhammer, CIP Medien, and Oxford University Press; and is involved in clinical trials undertaken by Shire and Viforpharma. DC declares grants and personal fees from Shire and Servier; personal fees from Eli Lilly, Novartis, and Oxford University Press; and grants from Vifor. $\mathrm{CH}$ is supported by the National Institute of Health Research (NIHR) Nottingham Biomedical Research Centre and the NIHR MindTech MedTech Co-operative. $\mathrm{CH}$ and ES are members of the National Institute for Health and Care Excellence (NICE) ADHD Guideline Group. AZ declares honoraria for participating in Advisory boards or Data Safety Monitory Boards for Eli Lilly, Otsuka, Lundbeck, Takeda, and EduPharma; royalties from Oxford University Press and Giunti OS; and research grants from Lundbeck, Roche, Shire, and Vifor. AC is supported by the NIHR Oxford Cognitive Health Clinical Research Facility. CDG declares no competing interests.

\section{References}

1. Cortese S, Adamo N, Del Giovane C, et al. Comparative efficacy and tolerability of medications for attention-deficit hyperactivity disorder in children, adolescents, and adults: a systematic review and network meta-analysis. Lancet Psychiatry 2018; 5: 727-38.

2. Cortese S, Adamo N, Mohr-Jensen C, et al. Comparative efficacy and tolerability of pharmacological interventions for attention-deficit/hyperactivity disorder in children, adolescents and adults: protocol for a systematic review and network meta-analysis. BMJ Open 2017; 7: e013967.

3. Mavridis D, Giannatsi M, Cipriani A, Salanti G. A primer on network meta-analysis with phasis on mental health. Evid Based Ment Health 2015; 18: 40-46.

4. Storeb $\varnothing$ OJ, Ramstad E, Krogh HB, et al. Methylphenidate for children and adolescents with attention deficit hyperactivity disorder (ADHD). Cochrane Database Syst Rev 2015; 11 : CD009885.

5. Cipriani A, Zhou X, Del Giovane C, et al. Comparative efficacy and tolerability of antidepressants for major depressive disorder in children and adolescents: a network metaanalysis. Lancet 2016; 388: 881-90.

6. Cipriani A, Furukawa TA, Salanti G, et al. Comparative efficacy and acceptability of 21 antidepressant drugs for the acute treatment of adults with major depressive disorder: a systematic review and network meta-analysis. Lancet 2018; 391: 1357-66. 